\title{
Geosmin Biosynthesis in Streptomyces avermitilis. Molecular Cloning, Expression, and Mechanistic Study of the Germacradienol/Geosmin Synthase
}

\author{
David E. Cane, Xiaofei He, Seiji Kobayashi, Satoshi Ōmura, Haruo Ikeda \\ This paper is submitted in honor of the late Professor Kenneth L. Rinehart, a pioneer in \\ the study of natural products and their biosynthesis.
}

Received: May 30, 2006 / Accepted: August 3, 2006

(C) Japan Antibiotics Research Association

\begin{abstract}
Geosmin (1) is responsible for the characteristic odor of moist soil. The Gram-positive soil bacterium Streptomyces avermitilis produces geosmin (1) as well as its precursor germacradienol (3). The $S$. avermitilis gene SAV2163 (geoA) is extremely similar to the $S$. coelicolor A3(2) SCO6073 gene that encodes a germacradienol/geosmin synthase. S. avermitilis mutants with a deleted geoA were unable to produce either germacradienol (3) or geosmin (1). Biosynthesis of both compounds was restored by introducing an intact geo $A$ gene into the mutants. Incubation of recombinant GeoA, encoded by the SAV2163 gene of S. avermitilis, with farnesyl diphosphate (2) in the presence of $\mathrm{Mg}^{2+}$ gave a mixture of $(4 S, 7 R)$-germacra-1(10)E,5E-diene-11-ol (3) (66\%), (7S)-germacrene D (4) (24\%), geosmin (1) (8\%), and a hydrocarbon, tentatively assigned the structure of octalin 5 (2\%). Incubation of this germacradienol/geosmin synthase with $\left[1,1-{ }^{2} \mathrm{H}_{2}\right]$ FPP (2a) gave geosmin- $\mathrm{d}_{1}$ (1a), as predicted. When recombinant GeoA from either S. avermitilis or S. coelicolor A3(2) was incubated with nerolidyl diphosphate (8), only the acyclic elimination products $\beta$-farnesene (10), $(Z)$ - $\alpha$-farnesene (11), and $(E)$ $\alpha$-farnesene (12) were formed, thereby ruling out nerolidyl diphosphate as an intermediate in the conversion of farnesyl diphosphate to geosmin, germacradienol, and germacrene D.
\end{abstract}

D. E. Cane (Corresponding author), X. He: Department of Chemistry, Brown University, Box H, Providence, Rhode Island 02912-9108 USA, E-mail: David_Cane@brown.edu

S. Ōmura: The Kitasato Institute, 9-1, Shirokane 5-chome,
Keywords sesquiterpene, biosynthesis, geosmin, Streptomyces avermitilis, Streptomyces coelicolor

\section{Introduction}

Geosmin (1) is a well-known, odoriferous metabolite produced by a wide variety of microorganisms, including Streptomyces, cyanobacteria, myxobacteria, and various fungi, as well as by higher plants such as liverworts and sugar beets [1 7]. Geosmin, with an especially low detection threshold of $10 \sim 100$ parts per trillion, is responsible for the characteristic odor of freshly turned earth and is associated with unpleasant off-flavors in water, wine, and fish $[8,9]$.

Streptomyces avermitilis (S. avermectinius is a junior homotypic synonym of $S$. avermitilis), an industrially important Gram-positive bacterium used for the production of the potent anthelminthic macrolide avermectin, also produces geosmin. The 9.03-Mb linear chromosome of $S$. avermitilis harbors genes encoding at least six apparent terpene synthases that are individually implicated in carotene, hopanoid, and sesquiterpene biosynthesis [10, 11]. Among the sesquiterpene synthases, the 2178-bp geo $A$ gene (SAV2163) encodes a putative protein of 725 amino acids (aa) with significant similarity to the $S$. coelicolor

Minato-ku, Tokyo 108-8642, Japan

S. Kobayashi, H. Ikeda: Kitasato Institute for Life Sciences, Kitasato University, 1-15-1, Kitasato, Sagamihara, Kanagawa 228-8555, Japan 


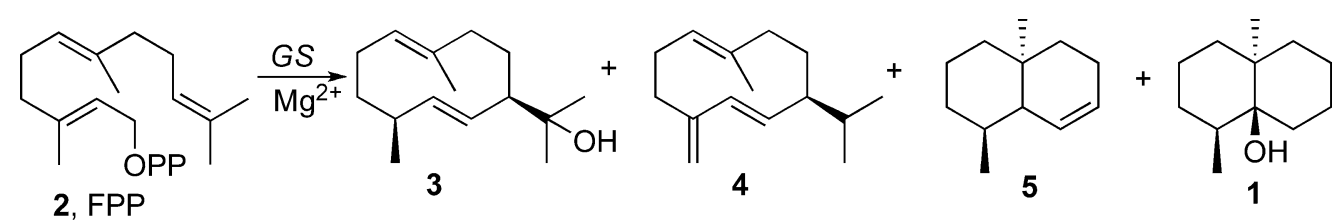

Scheme 1 Conversion of farnesyl diphosphate (FPP, 2) to geosmin (1).

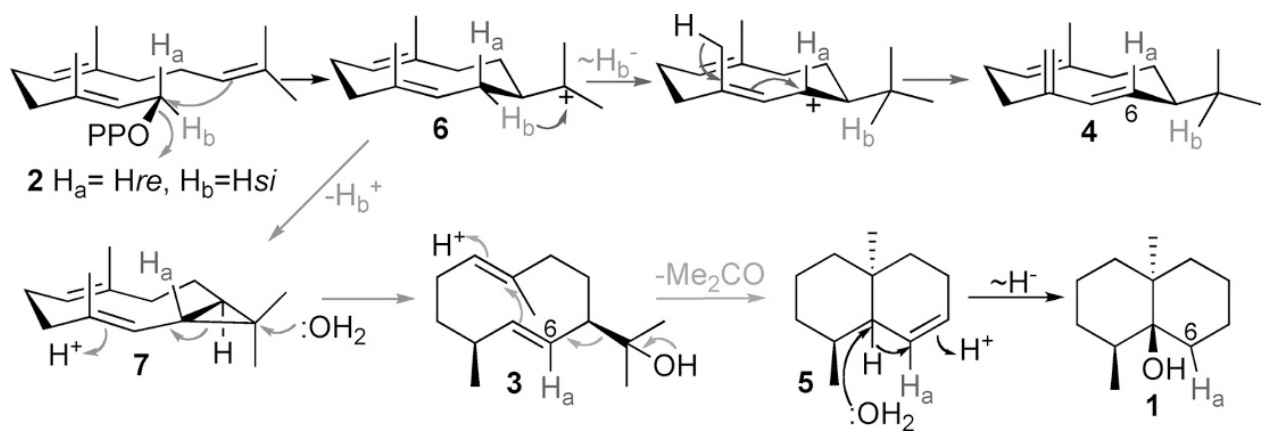

Scheme 2 Mechanism of the cyclization of FPP (2) to geosmin (1), germacradienol (3), germacrene D (4), and octalin $\mathbf{5}$.

A3(2) SCO6073 gene product [12]. The latter protein has been shown to catalyze the $\mathrm{Mg}^{2+}$-dependent conversion of farnesyl diphosphate (FPP, 2) to a mixture of $(4 S, 7 R)$ germacra-1(10)-diene-11-ol (3), germacrene $\mathrm{D}(4)$, a $\mathrm{C}_{12}$ hydrocarbon tentatively identified as octalin $\mathbf{5}$, and geosmin (1) (Scheme 1) [13 15].

The production of both germacradienol (3) and germacrene $\mathrm{D}$ (4) by the $S$. coelicolor A3(2) germacradienol/geosmin synthase has been shown to involve the partitioning of a common reaction intermediate, proposed to correspond to carbocation $\mathbf{6}$, in which loss of the original H-1si proton of FPP leads to formation of $\mathbf{3}$ while a competing 1,3-hydride shift of $\mathrm{H}-1$ si results in the generation of 4 (Scheme 2) [14]. The H-1re proton of FPP is retained at C-6 of both germacradienol and germacrene $\mathrm{D}$, as well as at (most likely) C-6 of geosmin. The relative proportion of geosmin to germacradienol in the product mixtures can be enhanced by increasing either the amount of enzyme or the incubation time, suggesting that germacradienol is transiently released from the cyclase and then rebound before conversion to geosmin [15].

Although the $N$ - and $C$-terminal halves of the $S$. coelicolor A3(2) germacradienol/geosmin synthase share a high level of sequence similarity, only the $N$-terminal domain catalyzes the conversion of FPP to germacradienol (2), while the $C$-terminal domain has no assigned biochemical function [13]. Molecular genetic experiments with $S$. coelicolor A3(2) deletion mutants have established that the $N$-terminal domain alone can support geosmin formation [14]. We now report the expression and biochemical characterization of the homologous $S$. avermitilis protein GeoA, and the confirmation that the recombinant enzyme is indeed a germacradienol/geosmin synthase. The function of the geoA gene product is also supported by the demonstration that a geoA deletion mutant does not produce either geosmin or germacradienol. Finally, we describe experiments that rule out the tertiary allylic isomer of FPP, nerolidyl diphosphate, as an intermediate in the enzymatic reaction.

\section{Experimental}

\section{General Procedures}

General analytical, molecular biological and biochemical methods were as previously described $[13,15,16]$. Recombinant germacradienol/germacrene D synthase, encoded by the SCO6073 gene of S. coelicolor A3(2) was expressed from E. coli BL21(DE3)pLysS/pRW31, resolubilized from inclusion bodies, purified to homogeneity, and assayed as previously described [13 15]. Farnesyl diphosphate, $\left[1,1-{ }^{2} \mathrm{H}_{2}\right]$ farnesyl diphosphate and nerolidyl diphosphate were each prepared as previously described $[17,18]$.

\section{GC-MS Analysis of Volatile Organic Products of S. avermitilis}

The spores of wild-type and mutants (see below) of $S$. avermitilis were used to inoculated a $100-\mathrm{ml}$ flask containing $10 \mathrm{ml}$ of vegetative medium (glucose $(0.5 \mathrm{~g})$, soy 
flour $(1.5 \mathrm{~g})$, and yeast extract $(0.5 \mathrm{~g})$ per $100 \mathrm{ml}, \mathrm{pH} 7.2)$ and the culture was allowed to grow while shaking at $30^{\circ} \mathrm{C}$ for 2 days. Geosmin production was observed in several media, but a synthetic medium was most suitable for the GC-MS analysis because many peaks were detected in the extract of the complex medium. A $0.1 \mathrm{ml}$ of portion of the culture was used to inoculate a $100-\mathrm{ml}$ flask containing $10 \mathrm{ml}$ of synthetic medium consisting of glucose $(60 \mathrm{~g})$, $\left(\mathrm{NH}_{4}\right)_{2} \mathrm{SO}_{4}(2 \mathrm{~g}), \mathrm{MgSO}_{4} \cdot 7 \mathrm{H}_{2} \mathrm{O}(0.1 \mathrm{~g}), \mathrm{K}_{2} \mathrm{HPO}_{4}(0.5 \mathrm{~g})$, $\mathrm{NaCl}(2 \mathrm{~g}), \mathrm{FeSO}_{4} \cdot 7 \mathrm{H}_{2} \mathrm{O}(0.05 \mathrm{~g}), \mathrm{ZnSO}_{4} \cdot 7 \mathrm{H}_{2} \mathrm{O}(0.05 \mathrm{~g})$, $\mathrm{MnSO}_{4} \cdot 4 \mathrm{H}_{2} \mathrm{O}(0.05 \mathrm{~g}), \mathrm{CaCO}_{3}(5 \mathrm{~g})$, and yeast extract $(2 \mathrm{~g})$ per liter, $\mathrm{pH}$ 7.0. After incubation while shaking at $28^{\circ} \mathrm{C}$ for 4 days, the culture was filtered. The supernatant was extracted with $1 \mathrm{ml}$ of $n$-hexane or pentane and the organic layer was dried over $\mathrm{Na}_{2} \mathrm{SO}_{4}$ and filtered through a $1-\mathrm{cm}$ column of $\mathrm{Na}_{2} \mathrm{SO}_{4}$ in a Pasteur pipette. A 1 to $5-\mu \mathrm{l}$ portion of the extract was analyzed by GC-MS (Shimadzu GC$17 \mathrm{~A}, 70 \mathrm{eV}$, EI, positive ion mode; $30 \mathrm{~m} \times 0.25 \mathrm{~mm}$ neutral bond- 5 capillary column ( $5 \%$ phenylmethylsilicon), using a temperature program of $50 \sim 280^{\circ} \mathrm{C}$, temperature gradient of $20^{\circ} \mathrm{C} /$ minute). Geosmin (1) and germacradienol (3) were identified by comparison with the spectra of the corresponding reference compounds in the NIST/EPA/NIH MS Library (2002 version).

\section{Molecular Cloning and Expression of Recombinant \\ $S$. avermitilis Construction of geoA-deletion Mutant}

Two segments were amplified by PCR from $S$. avermitilis cosmid CL_228_H03 (http://avermitilis.ls.kitasatou.ac.jp/). A segment upstream of geoA (nt 2,639,510 to $2,637,781$ ) was amplified using the forward (5'CTCGAGAAGCTTTGTCGTTGCCCGCGACCGTCAGCA- $\left.3^{\prime}\right)$ and reverse (5'-CTCGAGTCTAGACATGGCCGGGCCCTACCCAGGGCC-3') primers to introduce HindIII and $X b a \mathrm{I}$ restriction sites (bold), respectively. The forward (5'-CTCGAGTCTAGATGAGGGGGGGGACAGGCGGCTG-3') and reverse (5'-CTCGAGAAGCTTGCCGCTCAGGCCGAGCAGGTGCAT-3') primers were used for the amplification of a second segment downstream of geoA (nt 2,635,564 to 2,633,807), with introduction of $X b a \mathrm{I}$ and HindIII sites (bold), respectively. These two amplified DNA segments were digested with $H i n$ dIII and $X b a \mathrm{I}$ then digested segments were ligated together into HindIII-cut pKU250 and transformed into $E$. coli $\mathrm{DH} 5 \alpha$. The resultant plasmid was digested with $X b a \mathrm{I}$ and the linearized plasmid was ligated with the $X b a \mathrm{I}-$ fragment of the aad3" streptomycin/spectinomycin resistance cassette. The plasmid pKU250::upstream-aad3"downstream construct in E. coli recA $\mathrm{dcm} / \mathrm{pUB} 307$ was introduced into $S$. avermitilis by conjugation. After exoconjugants were selected by vector marker (thiostreptone resistance), geoA-deletion mutants, generated by double homologous recombination upstream and downstream of geoA, were obtained by selection for thiostreptone-sensitivity and streptomycin/spectinomycinresistance.

\section{Transfer of an Extra-copy of geoA Gene into S. avermitilis geoA-deletion Mutants}

Cosmid CL_228_H07 was digested with KpnI and the 4,594-bp segment (nt 2,634,026 to 2,638,620) carrying geoA was purified by agarose gel electrophoresis and ligated with the KpnI-cut $\phi \mathrm{C} 31$-based integrating vector carrying aphII as selectable marker. The resulting plasmid was used to transform $E$. coli $r e c A d c m / p U B 307:: T n 7$ and then transferred into the above-described $S$. avermitilis $\Delta$ geoA::aad3" by conjugation. The $S$. avermitilis $\triangle$ geoA::aad3"/geoA exoconjugants, were obtained by selection for neomycin resistance.

\section{Germacradienol/Geosmin Synthase (GeoA, SAV2163p)}

The geoA gene (SAV2163) was amplified by PCR from template DNA from $S$. avermitilis cosmid CL_228_H03 using the forward (5'-GGTAGGGAATTCCCATGACGCAGCCGTTCC- $\left.3^{\prime}\right)$ and reverse (5'-CTGTCCCTCGAGTCAGCGCGCCACC-3') primers to introduce EcoRI and XhoI restriction sites (bold) flanking the normal start and stop codons, respectively. The resulting amplicon and the pET21d(+) vector were digested separately with EcoRI and XhoI before ligation with T4 DNA ligase $(15: 1$ insert:vector, $4^{\circ} \mathrm{C}, 13$ hours) and transformation of electrocompetent $E$. coli XL-1 Blue cells. The resultant pXH17 plasmid was purified using a QIAprep Miniprep Kit and the sequence of the insert was confirmed by direct sequencing of both strands. (This construct appends the peptide MASMTGGQQMGRIRIP upstream of the native methionine start codon.) The purified pXH17 plasmid was then used to transform E. coli BL21(DE3) which was grown at $37^{\circ} \mathrm{C}$ in LB media $(400 \mathrm{ml}, \quad 260 \mathrm{rpm})$ supplemented with ampicillin $(100 \mathrm{mg} / \mathrm{liter})$ and chloramphenicol $(34 \mathrm{mg} / \mathrm{liter})$ to an $\mathrm{OD}_{600} \sim 0.3$, then induced by the addition of IPTG $(0.4 \mathrm{mM})$. After a further 5 hours at $30^{\circ} \mathrm{C}$, the cells were harvested by centrifugation. The cell pellet was resuspended in $40 \mathrm{ml}$ of lysis buffer (50 mM Tris $\cdot \mathrm{Cl}, 1 \mathrm{mM}$ EDTA, $20 \%$ glycerol (v/v), $\mathrm{pH} 8.2$ ). Lysozyme $(1.5 \mathrm{mg} / \mathrm{ml}), \quad \beta$-mercaptoethanol $(10 \mathrm{mM})$, benzamidine $(0.2 \mathrm{mM})$ and PMSF $(0.2 \mathrm{mM})$ were added to the cell suspension, which was then incubated at $34^{\circ} \mathrm{C}$, with shaking at $100 \mathrm{rpm}$ for 10 minutes. Benzonase ( $1 \mu \mathrm{l} /$ liter $)$, $\mathrm{MgCl}_{2}(10 \mathrm{mM})$ and Triton X-100 $(0.1 \%)$ were added and incubation was continued at $34^{\circ} \mathrm{C}$ for an additional 7 minutes. 
The insoluble fraction containing inclusion bodies was collected by centrifugation $(7500 \mathrm{~g}, 10$ minutes $)$, washed with $50 \mathrm{ml}$ of lysis buffer, and recentrifuged $(7500 \mathrm{~g}, 15$ minutes). The harvested inclusion bodies $(1.5 \mathrm{~g})$ were resuspended in $16 \mathrm{ml}$ of lysis buffer and added dropwise with stirring at $0^{\circ} \mathrm{C}$ to $200 \mathrm{ml}$ of $20 \mathrm{mM} \mathrm{NaOH}$ containing $5 \mathrm{mM} \beta$-mercaptoethanol and $0.02 \%$ (v/v) Triton X-100. After centrifugation to remove traces of residual insolubles $(15000 \mathrm{~g}, 10$ minutes), the basic solution was added at $8 \mathrm{ml} /$ minute with slow stirring at $4^{\circ} \mathrm{C}$ to 4 liters of refolding buffer (50 mM Tris $\cdot \mathrm{Cl}, 20 \%$ glycerol, $1 \mathrm{mM}$ EDTA, $5 \mathrm{mM}$ $\beta$-mercaptoethanol, $\mathrm{pH} 8.2$ ). The solution was allowed to equilibrate for 6 hours without further stirring, then adsorbed onto Q-Sepharose resin ( $70 \mathrm{ml}$, fast-flow) over 3 hours with stirring. The supernatant was decanted from the resin, which was then poured onto a column with a sintered filter, washed with refolding buffer containing $100 \mathrm{mM}$ $\mathrm{NaCl}$ (Buffer A) and then eluted with 1 liter of a linear gradient of $100 \mathrm{mM}$ to $500 \mathrm{mM} \mathrm{NaCl}$ in lysis buffer (Buffer B). Fractions were monitored by micro-Bradford assay [19] and SDS-PAGE and those containing the purest amounts of the desired protein $\left(M_{\mathrm{r}} \sim 83000\right)$ were combined, diluted 5.3-fold in Buffer A and loaded on a 50-ml Q-Sepharose (fast-flow) at $3 \mathrm{ml} /$ minute. After washing with $150 \mathrm{ml}$ of Buffer A, the column was eluted with 1.5 liters of a linear gradient of Buffer A to Buffer B. The purest fractions were pooled and concentrated by ultrafiltration to $3 \mathrm{ml}$ $(4.06 \mathrm{mg} / \mathrm{ml}$, based on uv at $280 \mathrm{~nm})$. The purified protein, which included a vector-derived hexadecapeptide upstream of the native $N$-terminal methionine, exhibited a single band $\left(M_{\mathrm{r}} \sim 83000\right)$ on SDS-PAGE, consistent with the predicted MW of $83402 \mathrm{Da}$.

\section{Assay of $S$. avermitilis Germacradienol/Geosmin Synthase (GeoA)}

The purified recombinant SAV2163 protein was assayed as previously described for the $S$. coelicolor germacradienol/geosmin synthase [13]. To determine the steady-state kinetic parameters, a series of assays was performed in $10-\mathrm{ml}$ glass tubes containing $1 \mathrm{ml}$ of assay buffer $\left(50 \mathrm{mM}\right.$ Tris $\cdot \mathrm{Cl}, 20 \%$ glycerol, $10 \mathrm{mM} \mathrm{MgCl}_{2}, \mathrm{pH}$ $8.2)$ and using a range of $\left[1-{ }^{3} \mathrm{H}\right]-\mathrm{FPP}$ concentrations $(8,16$, $40,100,200,300,500,750$, and $1000 \mathrm{nM}, 200 \mathrm{mCi} / \mathrm{mmol})$. The reactions, which were carried out in parallel, were initiated by the addition of $2 \mu 140$-fold diluted GeoA protein solution ( $200 \mathrm{ng}, 2.45 \mathrm{pmol}$ ), then immediately overlaid with $1.2 \mathrm{ml}$ of hexane. After 20 minutes incubation at $30^{\circ} \mathrm{C}$ (less than $10 \%$ conversion in all cases), the reaction was quenched by the addition of $75 \mu 1$ of $0.5 \mathrm{M}$ EDTA (pH 8.1). Control incubations utilized $40 \mathrm{nM}$ and $500 \mathrm{nM}$ [1$\left.{ }^{3} \mathrm{H}\right] \mathrm{FPP}$ in the absence of enzyme. Ether $(100 \mu \mathrm{l})$ was added and the mixture was vortexed for 30 seconds. The organic layer was passed through a $1-\mathrm{cm} \mathrm{SiO}_{2}$ column in a Pasteur pipette directly into a scintillation vial containing $7 \mathrm{ml}$ of Opti-Fluor. The aqueous layer was extracted with an additional $1.2 \mathrm{ml}$ of $11: 1$ hexane : ether which was added to the scintillation vial after passage through the $\mathrm{SiO}_{2}$ column which was then washed with $2 \times 0.75 \mathrm{ml}$ of ether. The steady-state kinetic parameters, $k_{\text {cat }}$ and $K_{\mathrm{m}}$, for the conversion of FPP to combined hydrocarbons and alcohols were calculated by direct fitting of the liquid scintillation data to the Michaelis-Menten equation by non-linear least squares regression using Kaleidagraph V3.6 (Adelbeck Software, Reading, PA, USA).

\section{Metal Ion Dependence}

To test divalent cation dependence of GeoA, a series of assays were carried out as above, replacing $\mathrm{MgCl}_{2}$ by $10 \mathrm{mM}$ concentrations of each of the following salts: $\mathrm{CaCl}_{2}$, $\mathrm{CoCl}_{2}, \mathrm{CuCl}_{2}, \mathrm{FeCl}_{2}, \mathrm{MnCl}_{2}, \mathrm{NiCl}_{2}$, and $\mathrm{ZnCl}_{2}$.

\section{Product Analysis}

Preparative scale incubations and GC-MS product analysis were carried out as previously described using $1 \mathrm{mg}$ $(12 \mathrm{nmol})$ of $S$. avermitilis germacradienol/geosmin synthase in $4.5 \mathrm{ml}$ of assay buffer containing $600 \mathrm{ng}$ $(270 \mathrm{nM})$ of FPP and overlaid with $3 \mathrm{ml}$ of HPLC-grade pentane [15]. The incubations were carried out for 20 minutes, 1 hour, and 3.5 hours at $30^{\circ} \mathrm{C}$ in $18 \times 150 \mathrm{~mm}$ glass tubes fitted with a rubber septum. After extraction of the reaction mixture with the pentane layer, the mixtures were extracted with an addition two $3-\mathrm{ml}$ portions of pentane. The combined organic layers were dried over $\mathrm{MgSO}_{4}$, filtered through a $2.5-\mathrm{cm}$ column of $\mathrm{MgSO}_{4}$ in a Pasteur pipette, and concentrated under reduced pressure at $0{ }^{\circ} \mathrm{C}$ to $\sim 100 \mu \mathrm{l}$. A $1-\mu \mathrm{l}$ portion of the concentrated extract was analyzed by GC-MS (JEOL JMS-600H, $70 \mathrm{eV}$, EI, positive ion mode; $30 \mathrm{~m} \times 0.25 \mathrm{~mm}$ HP5MS capillary column, using a temperature program of $60 \sim 280^{\circ} \mathrm{C}$, temperature gradient of $25^{\circ} \mathrm{C} /$ minute). The following products were detected for the 3.5-hours incubation: presumed octalin 5 (r.t. 5.18 minutes, $[\mathrm{M}]^{+} \mathrm{m} / z$ 164, base peak $\mathrm{m} / \mathrm{z}$ 149), geosmin (1) (r.t. 6.20 minutes, $[\mathrm{M}]^{+} \mathrm{m} / \mathrm{z}$ 182 , base peak $m / z$ 112), germacrene D (4) (r.t. 6.43 minutes, $\left[\mathrm{M}^{+}\right] \mathrm{m} / \mathrm{z}$ 204, base peak, $m / z$ 161), and germacradienol (3) (r.t. 7.29 minutes, $\left[\mathrm{M}^{+}\right] \mathrm{m} / \mathrm{z} 222$, base peak, $m / z$ 82), as well as a minor sesquiterpene hydrocarbonof asyet unassigned structure (r.t. 6.35 minutes, $\left[\mathrm{M}^{+}\right] \mathrm{m} / \mathrm{z} 204$, base peak, $m / z$ 107). Only germacradienol and germacrene $\mathrm{D}$ were detected in the 20 minutes incubation mixture. 


\section{Incubation with Nerolidyl Diphosphate}

Recombinant $S$. coelicolor germacradienol/geosmin synthase $(0.4 \mathrm{mg}, 4.8 \mathrm{nmol})$ was incubated as described above in $4.8 \mathrm{ml}$ of assay buffer containing $600 \mathrm{ng}(120 \mathrm{nM})$ of NPP (8) and overlaid with $3 \mathrm{ml}$ of HPLC-grade pentane for 3 hours. A $1-\mu 1$ portion of the concentrated pentane extract was analyzed by GC-MS (Hewlett-Packard Series 2 GC-MSD, $70 \mathrm{eV}$, EI, positive ion mode; $30 \mathrm{~m} \times 0.25 \mathrm{~mm}$ HP5MS capillary column, using a temperature program of $50 \sim 280^{\circ} \mathrm{C}$, temperature gradient of $20^{\circ} \mathrm{C} /$ minute and solvent delay of 4.0 minutes). The following products were detected: $\beta$-farnesene (10, r.t. 9.58 minutes, $[\mathrm{M}]^{+} \mathrm{m} / \mathrm{z} 204$, base peak $m / z 69),(3 Z)-\alpha$-farnesene (11, r.t. 9.78 minutes, $\left[\mathrm{M}^{+}\right] \mathrm{m} / \mathrm{z} 204$, base peak, $m / z$ 93); and (3E)- $\alpha$-farnesene (12, r.t. 9.88 minutes, $\left[\mathrm{M}^{+}\right] \mathrm{m} / \mathrm{z}$ 204, base peak, $m / z$ 93). Each compound was identified by comparison with the spectra of the corresponding reference compounds in the NIST/EPA/NIH MS Library (2002 version).

\section{Results}

Streptomyces avermitilis produces the typical strong odor of moist soil when grown in several different media. GCMS analysis of the $n$-hexane or pentane extract of a liquid culture revealed two main peaks (Fig. 1B), rt 9.89 minutes $\left[\mathrm{M}^{+}\right] \mathrm{m} / \mathrm{z} 182$ and 11.42 minutes $\left[\mathrm{M}^{+}+1\right] \mathrm{m} / \mathrm{z} 223$, that were identical to geosmin (1) and germacradienol, (3), respectively, by comparison with authentic samples of each compound (Fig. 1).

The $S$. avermitilis open-reading frame, geoA (SAV2163), encodes a protein with $77 \%$ identity and $87 \%$ similarity over 713 aa to the $S$. coelicolor A3(2) germacradienol/gesomin synthase (SCO6073p). Interestingly, the level of identity between the catalytically functional $\mathrm{N}$ terminal domains of the $S$. avermitilis and $S$. coelicolor A3(2) proteins is even greater: $89 \%$ identity and 98\% similarity over 327 aa. Both proteins harbor an aspartaterich ${ }^{84}$ DDHFLE motif and a downstream NSE triad

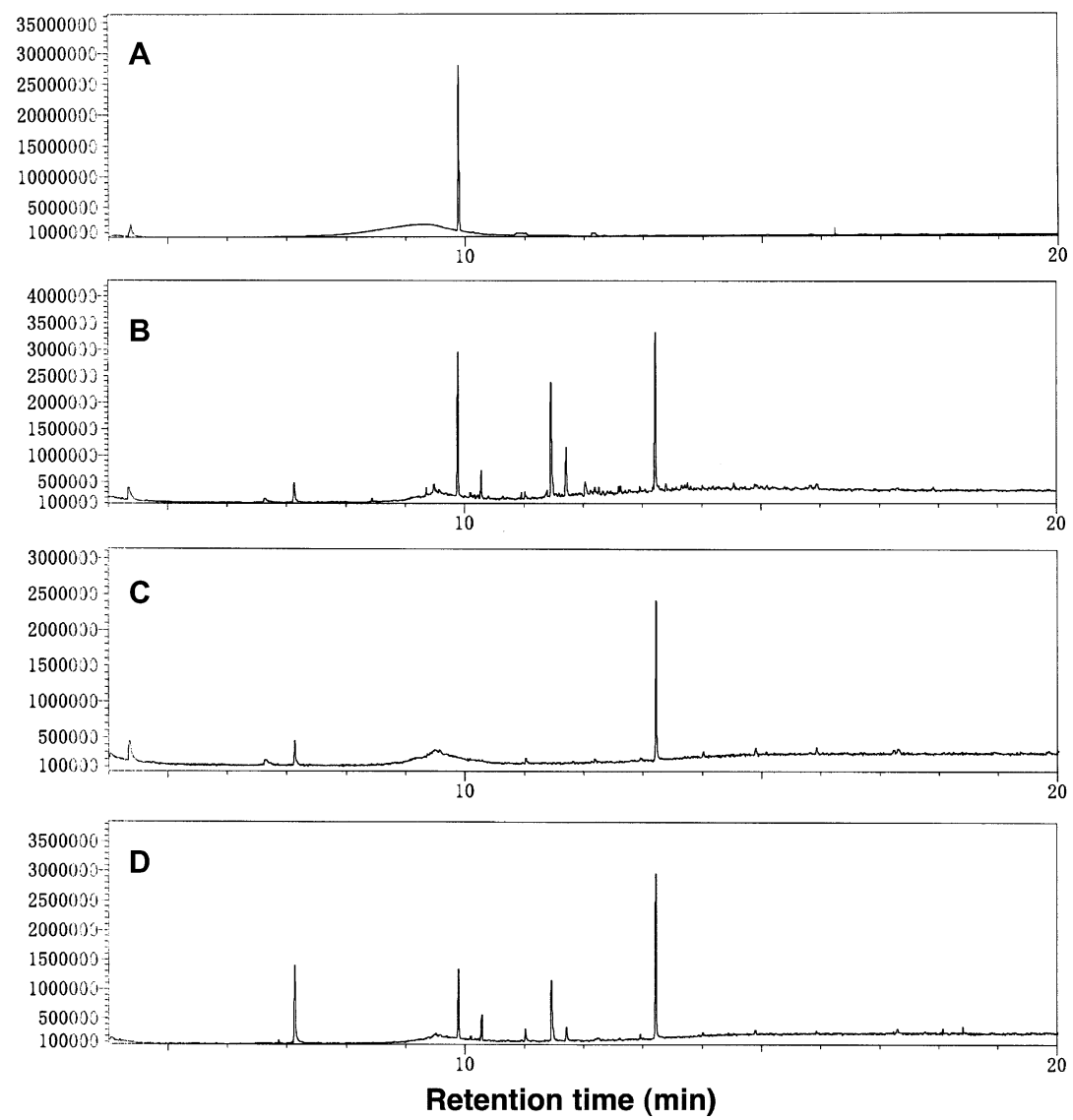

Fig. 1 GC-MS analysis of extracts from cultured broth of $S$. avermitilis and its mutants.

(A) Authentic sample of geosmin (1, rt 9.89 minutes, [M] ${ }^{+} \mathrm{m} / \mathrm{z}$ 182), (B) $n$-hexane extract from wild type strain of $S$. avermitilis. The peak at 11.42 minutes is germacradienol $\left(\mathbf{3},\left[\mathrm{M}^{+}+1\right] \mathrm{m} / z\right.$ 223). (C) $n$-hexane extract from $\Delta$ geoA::aad3" and (D) $n$-hexane extract from exoconjugant of geoA-deletion mutant carrying an extra-copy of geoA. (The peak at rt 13.30 minutes is diethyl phthalate $\left([\mathrm{M}]^{+} \mathrm{m} / z 222\right)$.) 
${ }^{224}$ NDLFSYQRE, the universally conserved $\mathrm{Mg}^{2+}$-binding domains that are found in all known sesquiterpene synthases.

We constructed a geoA-deletion mutant by homologous recombination in order to confirm the involvement of the GeoA in geosmin biosynthesis. Two 1.8-kb segments corresponding to both upstream and downstream regions of geo $A$ were amplified by PCR and ligated together into an $E$. coli/Streptomyces conjugative vector. A resistance gene (aad3") was inserted between these two amplified segments. After introducing the recombinant plasmid into wild-type $S$. avermitilis by selection of the vector marker, thiostreptone-resistance, homologous recombination between the chromosome and the recombinant plasmid gave the desired geoA-deletion mutants, which were obtained by selection for the thiostreptone-sensitive and streptomycin/spectinomycin resistance phenotype. The geoA deletion mutants failed to produce either geosmin (1) or germacradienol (3) (Fig. 1C). Introduction of an extra copy of wild-type geoA into these deletion mutants restored the production of both geosmin (1) and germacradienol (3) (Fig. 1D), indicating that GeoA is essential for the formation of both geosmin (1) and germacradienol (3).

We next used PCR to amplify the 2178-bp coding region of SAV2163, using DNA from $S$. avermitilis cosmid CL_228_H03 as template, while introducing EcoRI and $X h o \mathrm{I}$ restriction sites at the respective $5^{\prime}$ - and $3^{\prime}$-termini of the ORF. After ligation of the amplified DNA into the EcoRI/XhoI cloning sites of the pET21d(+) expression vector, the derived plasmid, pXH17, was purified from Escherichia coli XL-1 Blue and used to transform the T7 RNA polymerase-based expression host E. coli BL21(DE3). The resultant recombinant protein was obtained as insoluble inclusion bodies that were readily solubilized using $20 \mathrm{mM} \mathrm{NaOH}$ and purified to homogeneity, using a protocol previously developed for the homologous $S$. coelicolor A3(2) germacradienol/geosmin synthase [13]. A total of $12 \mathrm{mg}$ of purified recombinant GeoA (SAV2163p) was obtained from $400 \mathrm{ml}$ of culture.

Incubation of $2.5 \mathrm{nM}$ GeoA with $270 \mathrm{nM}$ FPP (2) for 3.5 hours at $30^{\circ} \mathrm{C}$ followed by GC-MS analysis of the pentanesoluble extract revealed the formation of germacradienol (3), germacrene D (4), octalin 5 , and geosmin (1) in a ratio of $66: 24: 2: 8$. The individual components were unambiguously identified by direct comparison of GCretention times and EI-mass spectra with those of authentic $\mathbf{3}, \mathbf{4}, \mathbf{5}$, and $\mathbf{1}$ that were generated from FPP in a separate incubation with recombinant $S$. coelicolor A3(2) germacradienol/geosmin synthase [15]. A small amount (3\%) of an additional, unidentified sesquiterpene hydrocarbon, $m / z$ 204, was also detected in the product mixture. As previously observed for the homologous $S$. coelicolor enzyme, no geosmin was detected after only 20 minutes incubation time, while both the absolute yield and the relative proportion of geosmin increased with longer incubation times. Incubation of $S$. avermitilis GeoA with $\left[1,1-{ }^{2} \mathrm{H}_{2}\right]$ FPP (2a) gave, as expected, germacradienol- $\mathrm{d}_{1}$ (3a) $([\mathrm{M}+1] \mathrm{m} / \mathrm{z} 223)$, germacrene $\mathrm{D}-\mathrm{d}_{2}(\mathbf{4 a})([\mathrm{M}+2] \mathrm{m} / \mathrm{z}$ 206, $\left[\mathrm{M}+2-\mathrm{C}_{3} \mathrm{H}_{6} \mathrm{D}\right] \mathrm{m} / z$ 162), and geosmin-d $\mathrm{d}_{1}$ (1a) $([\mathrm{M}+1] \mathrm{m} / \mathrm{z} 183$; base peak $m / z$ 113). As previously observed, the deuterium atom in $1 \mathbf{a}$ could be assigned to ring $\mathrm{B}$, as deduced from the characteristic MS fragment at $m / z 113\left(\mathrm{C}_{7} \mathrm{H}_{11} \mathrm{DO}\right)$ (Scheme 3) [15, 20].

The activity of recombinant GeoA, which was tested over a $\mathrm{pH}$ range from 5.4 9.5, showed optimal activity between $\mathrm{pH} 7.6$ and 9.0. The reaction showed an absolute requirement for a divalent cation, $\mathrm{Mg}^{2+}(10 \mathrm{mM})$ being preferred, with $\mathrm{Fe}^{2+}$ and $\mathrm{Zn}^{2+}$ exhibiting 50\% activity, and $\mathrm{Co}^{2+}, \mathrm{Cu}^{2+}$, or $\mathrm{Mn}^{2+}$ each showing $10 \sim 20 \%$ activity. Neither $\mathrm{Ca}^{2+}$ nor $\mathrm{Ni}^{2+}$ supported turnover of FPP. The steady-state kinetic parameters for recombinant GeoA were determined using $\left[1-{ }^{3} \mathrm{H}\right]$-FPP over a concentration range of $8 \mathrm{nM}$ to $2 \mu \mathrm{M}$. The calculated $k_{\text {cat }}$ for the formation of combined pentane soluble hydrocarbons and alcohols was $3.10 \pm 0.09 \times 10^{-3} \mathrm{~s}^{-1}$, with a $K_{\mathrm{m}}$ for FPP of $75 \pm 9 \mathrm{nM}$, similar to the $k_{\text {cat }}$ of $6.2 \pm 0.5 \times 10^{-3} \mathrm{~s}^{-1}$ and $K_{\mathrm{m}}$ of $62 \pm 8 \mathrm{nM}$ previously established for the recombinant $S$. coelicolor A3(2) enzyme [13].

The partitioning of a common cyclization intermediate in the formation of germacradienol (3) and germacrene D (4), involving a competition between loss of the original $\mathrm{H}-1$ si of FPP and a 1,3-hydride-shift, has important mechanistic and stereochemical implications. As illustrated in Scheme 2, direct cyclization of (E,E)-FPP followed by loss of H-1si would generate the trans-fused bicyclic intermediate, isolepidozene (7), the presumed precursor of

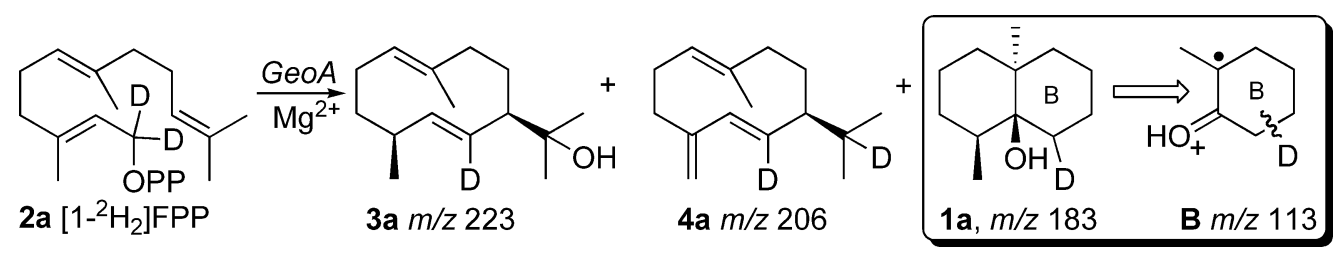

Scheme 3 Cyclization of $\left[1,1-{ }^{2} \mathrm{H}_{2}\right]$ FPP (2a) to geosmin (1a) and MS analysis. 


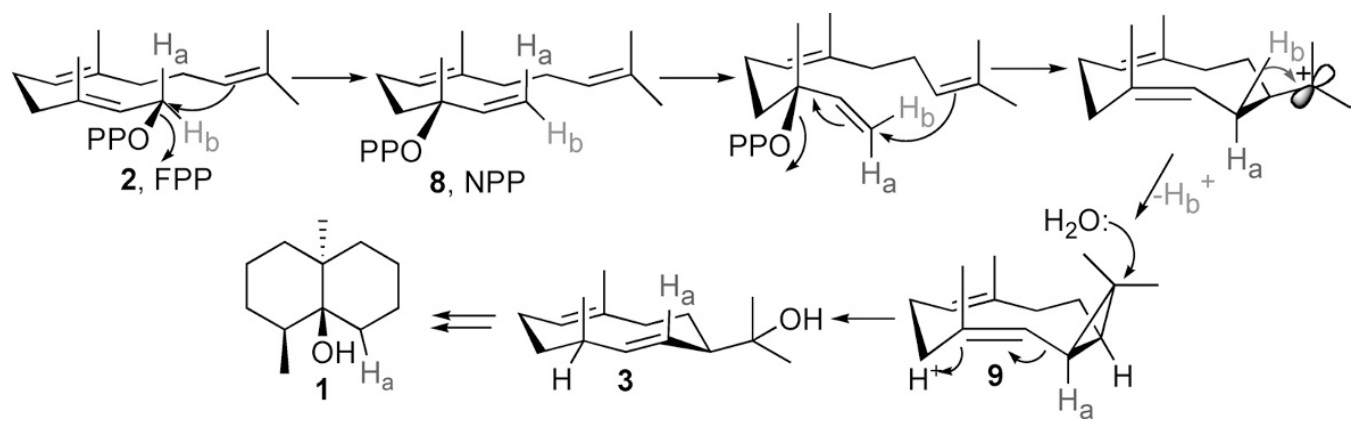

Scheme 4 Hypothetical intermediacy of nerolidyl diphosphate (8, NPP) and bicyclohelminthogermacrene (9) in geosmin formation.

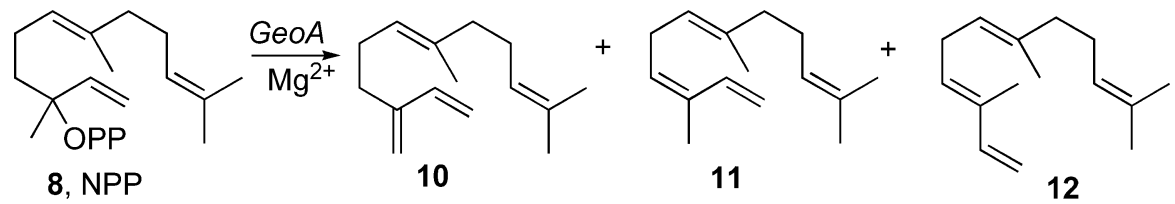

Scheme 5 Incubation of NPP (8) with germacradienol/geosmin synthase.

germacradienol, while prior isomerization to the tertiary allylic isomer, nerolidyl diphosphate (NPP, 8) would result in the intermediacy of the stereoisomeric, bicyclohelminthogermacrene (9) (Scheme 4) [14]. To distinguish between these two possibilities, we carried out separate incubations of NPP with $1 \mu \mathrm{M}$ recombinant germacradienol/geosmin synthases from both $S$. coelicolor $\mathrm{A} 3(2)$ and $S$. avermitilis and analyzed the resulting product mixtures by GC-MS. In place of $\mathbf{3}, \mathbf{4}, \mathbf{5}$, and $\mathbf{1}$, the normal products of FPP cyclization, the only compounds generated from NPP were the acyclic elimination products $\beta$ farnesene (10), (Z)- $\alpha$-farnesene (11), and ( $E$ )- $\alpha$-farnesene (12) (Scheme 5). Nerolidyl diphosphate is therefore firmly excluded as an intermediate in the enzymatic formation of germacradienol, germacrene $\mathrm{D}$, and geosmin.

\section{Discussion}

Germacradienol (3) was first identified in 1995 as a cometabolite of geosmin in S. citreus [4, 21]. Since that time, germacradienol has frequently been observed as a cometabolite of geosmin in streptomycetes, myxobacteria, and liverworts, often accompanied by germacrene D (4) [5, $6,22]$. Consistent with the demonstration that $S$. coelicolor A3(2) harbors a germacradienol synthase encoded by the SC06073 gene, and that this gene is essential for geosmin production, we and others originally proposed a variety of multistep biosynthetic pathways involving both oxidative and reductive transformations to account for the evident conversion of germacradienol to geosmin [4, 13, 20, 23]. Very recently, we have made the unexpected discovery that the protein encoded by SCO6073 can catalyze the entire conversion of FPP to geosmin itself, without requirement for any additional enzymes or redox cofactors [15]. This germacradienol/geosmin synthase is therefore both necessary and sufficient to support geosmin biosynthesis from FPP.

Both germacradienol (3) and geosmin (1) were found in the culture broth of $S$. avermitilis. The observed $\sim 5: 6$ ratio of germacradienol/geosmin was essentially the same in the several media examined. A geoA (SAV2163) deletion mutant constructed by homologous recombination produced neither geosmin nor germacradienol, while production of both compounds could be restored by complementation of the mutation by introducing an extra copy of the geo $A$ gene, confirming that GeoA is essential for geosmin biosynthesis.

We have established that the GeoA protein of $S$. avermitilis, encoded by geo $A$, which exhibits a high degree of sequence similarity to the $S$. coelicolor A3(2) enzyme SC06073p, catalyzes the predicted conversion of FPP to a mixture of germacradienol (3), germacrene D (4), and geosmin (1), as well as the presumed intermediate, octalin 5. Recombinant $S$. avermitilis GeoA has a product profile, divalent metal ion dependence, $\mathrm{pH}$ optimum, and steadystate kinetic parameters that are essentially the same as those previously determined for the $S$. coelicolor A3(2) germacradienol/geosmin synthase [15]. Moreover, incubation of recombinant $S$. avermitilis GeoA with [1,1- 
${ }^{2} \mathrm{H}_{2}$ ]-FPP gave deuterated germacradienol (3a), germacrene $\mathrm{D}$ (4a), and geosmin (1a) with labeling patterns that were identical to those previously obtained with the $S$. coelicolor A3(2) synthase [15]. Significantly, for both enzymes, the relative proportion of $\mathbf{1}$ with respect to germacradienol was enhanced by increasing the concentration of synthase or by employing longer incubation times. One would not expect such a result if both geosmin and germacradienol were to be generated by partitioning of a single series of enzymebound intermediates. Instead, it appears likely that a substantial fraction of the initially generated germacradienol is released into solution before being subsequently rebound and further converted to geosmin.

The finding that nerolidyl diphosphate is converted only to the acyclic farnesene isomers $\mathbf{1 0}, \mathbf{1 1}$, and $\mathbf{1 2}$ by both the $S$. avermitilis and the $S$. coelicolor A3(2) germacradienol/geosmin synthase rules out NPP as an intermediate in the conversion of FPP to germacradienol, germacrene D, and geosmin. Since the 2,3E-double bond of FPP therefore does not undergo geometric isomerization in the course of the enzyme-catalyzed cyclization, the established loss or migration of $\mathrm{H}-1 \mathrm{si}$ leads to the inference that the trans-fused bicyclogermacrene isomer, isolepidozene (7), would be the common sesquiterpene precursor of both germacradienol (3) and geosmin (1). Although we have not yet detected 7 as a product of the germacradienol/geosmin synthase reacton, (-)isolepidozene (7) has been reported as a metabolite of several liverworts, including Preissia quadrata [24]. It is also noteworthy that the corresponding alcohol, $\left(4 S^{*}, 5 S^{*}, 6 R^{*}, 7 R^{*}\right)$-1(10)E-lepidozen-5-ol (13) has been isolated from the liverwort Dumortiera hirsuta in which it is a cometabolite of germacradienol (3) (Fig. 2) [22]. Both alcohols compounds represent alternative hydration products of isolepidozene. Lepidozenol has also been isolated from the liverwort Trocholejeunea sandvicensis [25].

The finding that $S$. avermitilis GeoA converts farnesyl diphosphate to a mixture of germacradienol, germacrene D, and geosmin reinforces the recent discovery that the formation of geosmin from farnesyl diphosphate is mediated by a single enzyme. A similar geo $A$ open reading frame that shows $66 \%$ identity and $84 \%$ similarity over 718 aa to the S. avermitilis GeoA has also been found in the recently determined genomic sequence of $S$. peucetius (Prof. J. K. Sohng, personal communication) [26]. The mechanistic details of the remarkable reaction catalyzed by these geosmin synthases are currently under active investigation.

Acknowledgments This work was supported by NIH grant

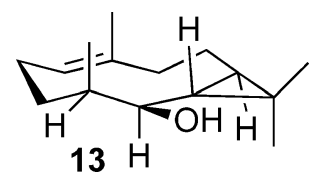

Fig. 2 Lepidozenol (13) isolated from the liverworts $D$. hirsuta and T. sandvicensis.

GM30301 to DEC, by Grant of the 21st Century COE Program, Ministry of Education, Culture, Sports, Science and Technology, Japan to H.I. and S.O., Grant-in-Aid for Scientific Research on Priority Areas from Ministry of Education, Culture, Sports, Science and Technology, Japan No. 17019009 to H.I., and by Grant-in-Aid for Scientific Research of the Japan Society for the Promotion of Science No. 17510168 to H.I. We would like to thank Jiaoyang Jiang for helpful advice in the preparation of the manuscript.

References

1. Gerber NN. Sesquiterpenoids from actinomycetes: Cadin-4ene-1-ol. Phytochemistry 10: 185-189 (1971)

2. Gerber NN, Lechevalier HA. Geosmin, an earthly-smelling substance isolated from actinomycetes. Appl Microbiol 13: 935-938 (1965)

3. Gerber NN. Geosmin, from microorganisms, is trans-1,10dimethyl-trans-9-decalol. Tetrahedron Lett 2971-2974 (1968)

4. Pollak FC, Berger RG. Geosmin and related volatiles in bioreactor-cultured Streptomyces citreus CBS 109.60. Appl Environ Microbiol 62: 1295-1299 (1996)

5. Dickschat JS, Wenzel SC, Bode HB, Muller R, Schulz S. Biosynthesis of volatiles by the myxobacterium Myxococcus xanthus. Chembiochem 5: 778-787 (2004)

6. Dickschat JS, Bode HB, Wenzel SC, Muller R, Schulz S. Biosynthesis and identification of volatiles released by the myxobacterium Stigmatella aurantiaca. Chembiochem 6: 2023-2033 (2005).

7. Lu G, Edwards CG, Fellman JK, Mattinson DS, Navazio J. Biosynthetic origin of geosmin in red beets (Beta vulgaris L.). J Agric Food Chem 51: 1026-1029 (2003).

8. Gerber NN. Volatile substances from actinomycetes: their role in the odor pollution of water. CRC Crit Rev Microbiol 7: 191-214 (1979)

9. La Guerche S, Chamont S, Blancard D, Dubourdieu D, Darriet P. Origin of (-)-geosmin on grapes: on the complementary action of two fungi, Botrytis cinerea and Penicillium expansum. Antonie Van Leeuwenhoek 88: 131-139 (2005)

10. Ikeda H, Ishikawa J, Hanamoto A, Shinose M, Kikuchi H, Shiba T, Sakaki Y, Hattori M, Ōmura S. Complete genome sequence and comparative analysis of the industrial microorganism Streptomyces avermitilis. Nat Biotechnol 21: 
526-531 (2003)

11. Ōmura S, Ikeda H, Ishikawa J, Hanamoto A, Takahashi C, Shinose M, Takahashi Y, Horikawa H, Nakazawa H, Osonoe T, Kikuchi H, Shiba T, Sakaki Y, Hattori M. Genome sequence of an industrial microorganism Streptomyces avermitilis: deducing the ability of producing secondary metabolites. Proc Natl Acad Sci USA 98: 12215-12220 (2001)

12. Gust B, Challis GL, Fowler K, Kieser T, Chater KF. PCRtargeted Streptomyces gene replacement identifies a protein domain needed for biosynthesis of the sesquiterpene soil odor geosmin. Proc Natl Acad Sci USA 100: 1541-1546 (2003)

13. Cane DE, Watt RM. Expression and mechanistic analysis of a germacradienol synthase from Streptomyces coelicolor implicated in geosmin biosynthesis. Proc Natl Acad Sci USA 100: 1547-1551 (2003)

14. He X, Cane DE. Mechanism and stereochemistry of the germacradienol/germacrene D synthase of Streptomyces coelicolor A3(2). J Am Chem Soc 126: 2678-2679 (2004)

15. Jiang J, He X, Cane DE. Geosmin Biosynthesis. Streptomyces coelicolor Germacradienol/Germacrene D synthase converts farnesyl diphosphate to geosmin. J Am Chem Soc 128: 8128-8129 (2006)

16. Tetzlaff CN, You Z, Cane DE, Takamatsu S, Ōmura S, Ikeda H. A gene cluster for biosynthesis of the sesquiterpenoid antibiotic pentalenolactone in Streptomyces avermitilis. Biochemistry 45: 6179-6186 (2006)

17. Cane DE, Oliver JS, Harrison PHM, Abell C, Hubbard BR, Kane CT, Lattman R. The biosynthesis of pentalenene and pentalenolactone. J Am Chem Soc 112: 4513-4524 (1990)
18. Cane DE, Chiu HT, Liang PH, Anderson KS. Pre-steadystate kinetic analysis of the trichodiene synthase reaction pathway. Biochemistry 36: 8332-8339 (1997)

19. Bradford M. A rapid and sensitive method for the quantitation of microgram quantitites of protein utilizing the principle of protein-dye binding. Anal Biochem 72: 248-254 (1976)

20. Dickschat JS, Bode HB, Mahmud T, Muller R, Schulz S. A novel type of geosmin biosynthesis in myxobacteria. J Org Chem 70: 5174-5182 (2005)

21. Gansser D, Pollak FC, Berger RG. A sesquiterpene alcohol from Streptomyces citreus CBS 109.60. J Nat Prod 58: 1790-1793 (1995)

22. Toyota M, Yoshida T, Matsunami J, Asakawa Y. Sesquiterpene and other constituents of the liverwort Dumortiera hirsuta. Phytochemistry 44: 293-298 (1997)

23. Spiteller D, Jux A, Piel J, Boland W. Feeding of $\left[5,5-{ }^{2} \mathrm{H}_{2}\right]-1-$ desoxy-D-xylulose and $\left[4,4,6,6,6-{ }^{2} \mathrm{H}_{5}\right]$-mevalolactone to a geosmin-producing Streptomyces sp. and Fossombronia pusilla. Phytochemistry 61: 827-834 (2002)

24. Hardt IH, Rieck A, Konig WA, Muhle H. Isolepidozene, a diastereomer of bicyclogermacrene, in some liverworts. Phytochemistry. 40: 605-606 (1995)

25. Tori M, Arbiyanti H, Taira Z, Asakawa Y. Terpenoids of the liverwort Frullanoides densifolia and Trocholejeuna sandvicensis. Phytochemistry 32: 335-348 (1993)

26. Parajuli N, Basnet DB, Chan Lee H, Sohng JK, Liou K. Genome analyses of Streptomyces peucetius ATCC 27952 for the identification and comparison of cytochrome P450 complement with other Streptomyces. Arch Biochem Biophys 425: 233-241 (2004) 